\title{
STABILITY OF THE DIFFERENCE SCHEMES FOR THE EQUATIONS OF WEAKLY COMPRESSIBLE LIQUID
}

\author{
P. MATUS ${ }^{1}$, O. KOROLYOVA ${ }^{1}$, AND M. CHUIKO ${ }^{1}$
}

\begin{abstract}
A priory estimates of the stability in the sense of the initial data of the difference scheme approximating weakly compressible liquid equations in the Riemann invariants have been obtained. These estimates have been proved without any assumptions about the properties of the solution of the differential problem and depend only on the behavior of the initial conditions. As distinct from linear problems, the obtained estimates of stability in the general case exist only for a finite instant of time $t \leqslant t_{0}$. In particular, this is confirmed by the fact, that nonfulfilment of these stability conditions lead to the appearance of supersonic flows or domains with large gradients. The questions of uniqueness and convergence of the difference solution are considered also. The results of the computating experiment confirming the theoretical conclusions are given.
\end{abstract}

2000 Mathematics Subject Classification: 65M06; 65M12.

Keywords: stability, difference scheme, Riemann invariants, weakly compressible liquid.

\section{Introduction}

In investigating the difference schemes, primary consideration is given to the fundamental question of stability of the difference solution in the sense of the small perturbations of the input data. By now, the most complete results have been obtained for computational methods approximating the linear problems of mathematical physics [1,2].

The fundamental difference of the investigation of stability in the nonlinear case is due to the necessity of additionally investigating the properties of all difference derivatives entering into the nonlinear part of the difference equations. In addition, we don't impose any limitations on the behavior of the nonlinear coefficients of the original difference problem, except for the limitation on the initial data, when all parameters of the nonlinear problem are known. In nonlinear problems, such irregularities as shocks, boundary layers, etc. can arise independently of the input data smoothness. The first rigorous results of investigating the stability of the difference schemes for the nonlinear transfer equation, the Burgers equation, and the quasilinear parabolic equation were obtained in $[3,4]$.

The aim of the present work is to investigate the stability in the sense of the initial data of the difference scheme approximating the equations of a weakly compressible liquid in the Riemann invariants.

\footnotetext{
${ }^{1}$ Institute of Mathematics, NAS of Belarus, 11 Surganov Str., 220072 Minsk, Belarus. E-mail: matus@im.bas-net.by, korolyova@im.bas-net.by, chuiko@im.bas-net.by
} 
The conditions imposed on the initial velocity and pressure that guarantee subsonic flows have been obtained. The computating experiment has confirmed the theoretical conclusions that the violation of the conditions obtained results in the appearance of supersonic flows or an ill-posed statement of the boundary conditions.

This paper is organized as follows: Section 2 is devoted to the initial boundary value problem definition for the system of weakly compressible liquid equations. For the system of equations in the Riemann invariants the linearized difference scheme is used. The conditions for the initial data that guarantee stability of the difference scheme are introduced. In Section 3, auxiliary results for the proof of the stability are obtained. In Section 4, the stability of the difference scheme is investigated. A series of stability theorems are proved. The uniqueness of the solution of the difference scheme is studied. Section 5 is devoted to the investigation of the convergence of the difference scheme. Section 6 presents the results of the numerical experiment. In the last section, some conclusions are given.

\section{Problem statement}

In the rectangle $(x, t) \in \bar{Q}_{T}, \bar{Q}_{T}=\bar{\Omega} \times[0, T], \bar{\Omega}=\{x: 0 \leqslant x \leqslant l\}$, we consider the following initial boundary value problem for the system of weakly compressible liquid [5]:

$$
\begin{gathered}
\frac{\partial v}{\partial t}+v \frac{\partial v}{\partial x}+\frac{1}{\rho} \frac{\partial p}{\partial x}=0, \quad \frac{\partial p}{\partial t}+\rho c^{2} \frac{\partial v}{\partial x}+v \frac{\partial p}{\partial x}=0, \quad(x, t) \in \bar{Q}_{T}, \\
v(x, 0)=v_{0}(x), \quad p(x, 0)=p_{0}(x), \quad 0 \leqslant x \leqslant l, \\
p(0, t)+\rho c v(0, t)=\mu_{1}(t), \quad p(l, t)-\rho c v(l, t)=\mu_{2}(t), \quad 0<t \leqslant T .
\end{gathered}
$$

Here $v=v(x, t), p=p(x, t)$ are the pressure and velocity respectively, $\rho=$ const $>0$ is the liquid density, $c=$ const $>0$ is the sonic speed.

Let us define the vector $\vec{u}=(v, p)^{\top}$ and the matrix $A=\left\{a_{i j}\right\}_{i, j=1}^{2}$ with components $a_{11}=a_{22}=v, a_{12}=1 / \rho, a_{21}=\rho c^{2}$. According to [5], system (2.1) can be written in the canonical form of the first-order systems of differential equations

$$
\frac{\partial \vec{u}}{\partial t}+A \frac{\partial \vec{u}}{\partial x}=0
$$

The eigenvalues $\lambda_{1,2}=v \pm c$ of the matrix $A$ are found from the characteristic equation $|A-\lambda E|=0$. Since the eigenvalues $\lambda_{1}, \lambda_{2}$ are real and different, the system of equations (2.1) is hyperbolic. According to [5], the boundary conditions (2.3) will be set correctly only for subsonic flows $|v|<c,(x, t) \in \bar{Q}_{T}$.

Suppose, in addition, that the initial conditions (2.2) satisfy the inequalities

$$
\begin{gathered}
\left\|v_{0}\right\|_{C_{[0, l]}}+\frac{1}{\rho c}\left\|p_{0}\right\|_{C_{[0, l]}}<c, \\
0 \leqslant p_{0}^{\prime}(x)+\rho c v_{0}^{\prime}(x) \leqslant c_{0}, \quad-c_{1} \leqslant p_{0}^{\prime}(x)-\rho c v_{0}^{\prime}(x) \leqslant 0,
\end{gathered}
$$

where $\|f\|_{C_{[0, l]}}=\max _{x \in[0, l]}|f(x)|$. It will be shown below that (2.4) is the necessary condition for the well-posed statement of problem $(2.1)-(2.3)$.

Taking into account the Riemann invariants [7] $r=p+\rho c v, s=p-\rho c v$, we write the differential problem $(2.1)-(2.3)$ in the equivalent form

$$
\frac{\partial r}{\partial t}+(v+c) \frac{\partial r}{\partial x}=0, \quad \frac{\partial s}{\partial t}+(v-c) \frac{\partial s}{\partial x}=0,
$$




$$
\begin{array}{cc}
r(x, 0)=r_{0}(x)=p_{0}(x)+\rho c v_{0}(x), & s(x, 0)=s_{0}(x)=p_{0}(x)-\rho c v_{0}(x), \\
r(0, t)=\mu_{1}(t), & s(l, t)=\mu_{2}(t) .
\end{array}
$$

In the domain $\bar{Q}_{T}$, we introduce uniform space-time grid $\bar{\omega}=\bar{\omega}_{h} \times \bar{\omega}_{\tau}: \bar{\omega}_{h}=\left\{x_{i}: x_{i}=i h\right.$, $i=\overline{0, N}, h N=l\}, \bar{\omega}_{\tau}=\left\{t_{n}: t_{n}=n \tau, n=\overline{0, N_{0}}, \tau N_{0}=T\right\}=\omega_{\tau} \cup\{0\}$ with constant steps $h$ and $\tau$ in both directions. Hereinafter, the usual notations of the difference scheme theory are used [1]:

$$
y=y_{i}^{n}=y\left(x_{i}, t_{n}\right), \hat{y}=y_{i}^{n+1}=y\left(x_{i}, t_{n+1}\right), y_{\bar{x}, i}=\frac{y_{i}-y_{i-1}}{h}, y_{x, i}=\frac{y_{i+1}-y_{i}}{h}, y_{t, i}=\frac{\hat{y}_{i}-y_{i}}{\tau} .
$$

On the grid $\bar{\omega}$ the differential problem in the Riemann invariants $(2.6)-(2.8)$ is approximated by the linearized difference scheme

$$
\begin{gathered}
r_{h t, i}+\left(v_{h, i}+c\right) \hat{r}_{h \bar{x}, i}=0, \quad i=\overline{1, N}, \quad s_{h t, i}+\left(v_{h, i}-c\right) \hat{s}_{h x, i}=0, \quad i=\overline{0, N-1}, \\
r_{h, i}^{0}=p_{0, i}+\rho c v_{0, i}, \quad s_{h, i}^{0}=p_{0, i}-\rho c v_{0, i}, \quad i=\overline{0, N} \\
\hat{r}_{h, 0}=\mu_{1}\left(t_{n+1}\right), \quad \hat{s}_{h, N}=\mu_{2}\left(t_{n+1}\right), \quad n=\overline{0, N_{0}-1},
\end{gathered}
$$

where the invariants $r_{h, i}^{n+1}$ are determined consecutively from left to right and the invariants $s_{h, i}^{n+1}$ are determined from right to left by explicit formula. Here $v_{h}=\left(r_{h}-s_{h}\right) / 2 \rho c, p_{h}=$ $\left(r_{h}+s_{h}\right) / 2$.

\section{Auxiliary results}

In investigating the stability of the difference problem (2.9)-(2.11), we will use the following canonical forms of the two-point difference schemes for the first boundary value problem:

$$
\begin{aligned}
A_{i} y_{i-1}-C_{i} y_{i} & =-F_{i}, \quad i=\overline{i_{0}+1, i_{N}}, \quad y_{i_{0}}=\mu_{1}, \\
-C_{i} y_{i}+B_{i} y_{i+1} & =-F_{i}, \quad i=\overline{i_{0}, i_{N}-1}, \quad y_{i_{N}}=\mu_{2} .
\end{aligned}
$$

Lemma 3.1. Let the conditions $A_{i} \geqslant 0, D_{i}=C_{i}-A_{i}>0$ be met. Then for the solution of problem (3.1) the estimate

$$
\max _{i_{0} \leqslant i \leqslant i_{N}}\left|y_{i}\right| \leqslant \max \left\{\left|\mu_{1}\right|, \max _{i_{0}<i \leqslant i_{N}} \frac{\left|F_{i}\right|}{D_{i}}\right\}
$$

is valid.

Proof. Let $\left|y_{i}\right|$ reach the maximum value $\left|y_{i^{*}}\right|>0$ for $i=i^{*}, i_{0}<i^{*} \leqslant i_{N}$, so as $\left|y_{i^{*}}\right| \geqslant\left|y_{i}\right|$ for $i=\overline{i_{0}, i_{N}}$. Then from equation (3.1) for $i=i^{*}$

$$
C_{i^{*}}\left|y_{i^{*}}\right|=\left|C_{i^{*}} y_{i^{*}}\right| \leqslant A_{i^{*}}\left|y_{i^{*}-1}\right|+\left|F_{i^{*}}\right| \leqslant A_{i^{*}}\left|y_{i^{*}}\right|+\left|F_{i^{*}}\right|
$$

follows. Hence we define $\left(C_{i^{*}}-A_{i^{*}}\right)\left|y_{i^{*}}\right|=D_{i^{*}}\left|y_{i^{*}}\right| \leqslant\left|F_{i^{*}}\right|$ and, therefore,

$$
\max _{i_{0} \leqslant i \leqslant i_{N}}\left|y_{i}\right|=\left|y_{i^{*}}\right| \leqslant \frac{\left|F_{i^{*}}\right|}{D_{i^{*}}} \leqslant \max _{i_{0}<i \leqslant i_{N}} \frac{\left|F_{i}\right|}{D_{i}} .
$$

If $\left|y_{i}\right|$ reaches maximum for $i=i_{0}$, then $\max _{i_{0} \leqslant i \leqslant i_{N}}\left|y_{i}\right|=\left|y_{i_{0}}\right|=\left|\mu_{1}\right|$. Combining the two inequalities, we obtain the required estimate. 
Lemma 3.2. Let the conditions $B_{i} \geqslant 0, D_{i}=C_{i}-B_{i}>0$ be met. Then for the solution of problem (3.2) the estimate

$$
\max _{i_{0} \leqslant i \leqslant i_{N}}\left|y_{i}\right| \leqslant \max \left\{\left|\mu_{2}\right|, \max _{i_{0} \leqslant i<i_{N}} \frac{\left|F_{i}\right|}{D_{i}}\right\}
$$

is valid.

The proof of Lemma 3.2 is similar to the proof of Lemma 3.1.

Lemma 3.3 [3]. Let the conditions

$$
1-t_{n+1} a_{0}>0, \quad a_{n} \geqslant 0, \quad t_{n}=n \tau, \quad n=0,1, \ldots N_{0}, \quad \tau N_{0}=T
$$

be met. Then from the inequalities

$$
a_{n} \leqslant \frac{a_{n-1}}{1-\tau a_{n-1}}, \quad n=1,2, \ldots N_{0}
$$

the estimates

$$
a_{n} \leqslant \frac{a_{0}}{1-t_{n} a_{0}}, \quad 1-\tau a_{n}>0, \quad n=0,1, \ldots N_{0}
$$

follow.

\section{Stability investigation}

Along with problem (2.9) - (2.11), we consider the perturbed problem

$$
\begin{gathered}
\tilde{r}_{h t, i}+\left(\tilde{v}_{h, i}+c\right) \hat{\tilde{r}}_{h \bar{x}, i}=0, \quad i=\overline{1, N}, \quad \tilde{s}_{h t, i}+\left(\tilde{v}_{h, i}-c\right) \hat{\tilde{s}}_{h x, i}=0, \quad i=\overline{0, N-1}, \\
\tilde{r}_{h, i}^{0}=\tilde{p}_{0, i}+\rho c \tilde{v}_{0, i}, \quad \tilde{s}_{h, i}^{0}=\tilde{p}_{0, i}-\rho c \tilde{v}_{0, i}, \quad i=\overline{0, N} \\
\hat{\tilde{r}}_{h, 0}=\mu_{1}\left(t_{n+1}\right), \quad \hat{\tilde{s}}_{h, N}=\mu_{2}\left(t_{n+1}\right), \quad n=\overline{0, N_{0}-1 .}
\end{gathered}
$$

Let for the perturbed initial data $\tilde{v}_{0}, \tilde{p}_{0}$ the inequalities

$$
\begin{gathered}
\left\|\tilde{v}_{0}\right\|_{C_{[0, l]}}+\frac{1}{\rho c}\left\|\tilde{p}_{0}\right\|_{C_{[0, l]}}<c \\
0 \leqslant\left(\tilde{p}_{0}(x)\right)^{\prime}+\rho c\left(\tilde{v}_{0}(x)\right)^{\prime} \leqslant c_{0}, \quad-c_{1} \leqslant\left(\tilde{p}_{0}(x)\right)^{\prime}-\rho c\left(\tilde{v}_{0}(x)\right)^{\prime} \leqslant 0,
\end{gathered}
$$

similar to inequalities (2.4), (2.5) hold.

Subtracting the corresponding equations (2.9) - (2.11) from equations (4.1)-(4.3), we obtain the problem for the perturbations $\delta r_{i}=\tilde{r}_{h, i}-r_{h, i}, \delta s_{i}=\tilde{s}_{h, i}-s_{h, i}$

$$
\begin{gathered}
\delta r_{t, i}+\left(v_{h, i}+c\right) \delta \hat{r}_{\bar{x}, i}+\hat{\tilde{r}}_{h \bar{x}, i} \frac{\delta r_{i}}{2 \rho c}-\hat{\tilde{r}}_{h \bar{x}, i} \frac{\delta s_{i}}{2 \rho c}=0, \quad i=\overline{1, N}, \\
\delta s_{t, i}+\left(v_{h, i}-c\right) \delta \hat{s}_{x, i}+\hat{\tilde{s}}_{h x, i} \frac{\delta r_{i}}{2 \rho c}-\hat{\tilde{s}}_{h x, i} \frac{\delta s_{i}}{2 \rho c}=0, \quad i=\overline{0, N-1}, \\
\delta \hat{r}_{0}=0, \quad \delta \hat{s}_{N}=0, \quad \delta r_{i}^{0}=\delta p_{0, i}+\rho c \delta v_{0, i}, \quad \delta s_{i}^{0}=\delta p_{0, i}-\rho c \delta v_{0, i}, \quad i=\overline{1, N} .
\end{gathered}
$$

Hereinafter we will use the following grid norms:

$$
\left\|y_{h}\right\|_{C^{+}}=\max _{1 \leqslant i \leqslant N}\left|y_{h, i}\right|, \quad\left\|y_{h}\right\|_{C^{-}}=\max _{0 \leqslant i \leqslant N-1}\left|y_{h, i}\right|, \quad\left\|y_{h}\right\|_{\bar{C}}=\max _{0 \leqslant i \leqslant N}\left|y_{h, i}\right| .
$$


Definition 4.1. The difference scheme $(2.9)-(2.11)$ is absolutely stable in the sense of the initial data with respect to the uniform norm, if independent of the choice of $\tau, h$ and the initial data $p_{0}, \tilde{p}_{0}, v_{0}, \tilde{v}_{0}$, the constant $M>0$ exists and satisfies the inequality

$$
\left\|\tilde{r}_{h}^{n}-r_{h}^{n}\right\|_{\bar{C}}+\left\|\tilde{s}_{h}^{n}-s_{h}^{n}\right\|_{\bar{C}} \leqslant M\left(\left\|\tilde{r}_{h}^{0}-r_{h}^{0}\right\|+\left\|\tilde{s}_{h}^{0}-s_{h}^{0}\right\|_{\bar{C}}\right)
$$

for arbitrary $\tau, h$ and for all $t_{n} \in \bar{\omega}_{\tau}$.

For simplicity of the further investigations, we suppose that the boundary conditions are homogeneous, i.e., $\mu_{1}(t)=\mu_{2}(t)=0, \quad 0<t \leqslant T$. From the problem for perturbations $\delta r_{i}, \quad \delta s_{i}$ it follows that for obtaining a priory estimate (4.9) the difference derivatives $\tilde{r}_{h \bar{x}}, \quad \tilde{s}_{h x}$ have to be estimated. Using the technique of stability investigation for nonlinear difference schemes proposed in [2], it is possible to prove the following statements.

Theorem 4.1. Let condition (2.4) be met. Then for the solution of the difference problem (2.9)-(2.11) the estimates

$$
\left\|r_{h}^{n}\right\|_{\bar{C}} \leqslant\left\|p_{0}+\rho c v_{0}\right\|_{\bar{C}}, \quad\left\|s_{h}^{n}\right\|_{\bar{C}} \leqslant\left\|p_{0}-\rho c v_{0}\right\|_{\bar{C}}, \quad\left\|v_{h}^{n}\right\|_{\bar{C}}<c, n=\overline{0, N_{0}}
$$

are valid.

Proof. We write the difference scheme (2.9) - (2.11) in the canonical form

$$
\begin{gathered}
A_{r, i}^{n} r_{h, i-1}^{n+1}-C_{r, i}^{n} r_{h, i}^{n+1}=-F_{r, i}^{n}, \quad i=\overline{1, N}, \quad r_{h, 0}^{n+1}=\mu_{1}=0, \\
-C_{s, i}^{n} s_{h, i}^{n+1}+B_{s, i}^{n} s_{h, i+1}^{n+1}=-F_{s, i}^{n}, \quad i=\overline{0, N-1}, \quad s_{h, N}^{n+1}=\mu_{2}=0,
\end{gathered}
$$

where $A_{r, i}^{n}=\gamma\left(v_{h, i}^{n}+c\right), C_{r, i}^{n}=1+A_{r, i}^{n}, F_{r, i}^{n}=r_{h, i}^{n}, B_{s, i}^{n}=-\gamma\left(v_{h, i}^{n}-c\right), C_{s, i}^{n}=1+B_{s, i}^{n}$, $F_{s, i}^{n}=s_{h, i}^{n}, \gamma=\tau / h$.

We will use the method of induction. From condition (2.4) $\left\|v_{0}\right\|_{\bar{C}}<c$ follows. Then on the first time layer $t=\tau$ we obtain $A_{r, i}^{0}=\gamma\left(v_{0, i}+c\right)>0, C_{r, i}^{0}=1+A_{r, i}^{0}>0, F_{r, i}^{0}=r_{h, i}^{0}$, $B_{s, i}^{0}=-\gamma\left(v_{0, i}-c\right)>0, C_{s, i}^{0}=1+B_{s, i}^{0}>0, F_{s, i}^{0}=s_{h, i}^{0}$. On the basis of Lemmas 3.1, 3.2 we get $\left\|r_{h}^{1}\right\|_{\bar{C}} \leqslant\left\|r_{h}^{0}\right\|_{\bar{C}},\left\|s_{h}^{1}\right\|_{\bar{C}} \leqslant\left\|s_{h}^{0}\right\|_{\bar{C}}$. Then for the velocity grid function we obtain

$$
\left\|v_{h}^{1}\right\|_{\bar{C}} \leqslant \frac{\left\|r_{h}^{1}\right\|_{\bar{C}}+\left\|s_{h}^{1}\right\|_{\bar{C}}}{2 \rho c} \leqslant \frac{\left\|r_{h}^{0}\right\|_{\bar{C}}+\left\|s_{h}^{0}\right\|_{\bar{C}}}{2 \rho c} \leqslant\left\|v_{h}^{0}\right\|_{\bar{C}}+\frac{\left\|p_{h}^{0}\right\|_{\bar{C}}}{\rho c}<c .
$$

Now let us suppose that on the time layer $n$ the condition $\left\|v_{h}^{n}\right\|_{\bar{C}}<c$ is fulfilled. Prove that on the $(n+1)$ time layer $\left\|v_{h}^{n+1}\right\|_{\bar{C}}<c$. In fact from the induction assumption it follows that $A_{r, i}^{n}>0, C_{r, i}^{n}=1+A_{r, i}^{n}, B_{s, i}^{n}>0, C_{s, i}^{n}=1+B_{s, i}^{n}$. Hence all conditions of Lemmas 3.1, 3.2 are fulfilled and the inequalities $\left\|r_{h}^{n+1}\right\|_{\bar{C}} \leqslant\left\|r_{h}^{n}\right\|_{\bar{C}},\left\|s_{h}^{n+1}\right\|_{\bar{C}} \leqslant\left\|s_{h}^{n}\right\|_{\bar{C}}$ hold. Therefore, $\left\|r_{h}^{n+1}\right\|_{\bar{C}} \leqslant$ $\left\|r_{h}^{n}\right\|_{\bar{C}} \leqslant \ldots \leqslant\left\|r_{h}^{0}\right\|_{\bar{C}}=\left\|p_{0}+\rho c v_{0}\right\|_{\bar{C}},\left\|s_{h}^{n+1}\right\|_{\bar{C}} \leqslant\left\|s_{h}^{n}\right\|_{\bar{C}} \leqslant \ldots \leqslant\left\|s_{h}^{0}\right\|_{\bar{C}}=\left\|p_{0}-\rho c v_{0}\right\|_{\bar{C}}$. For the velocity grid function the inequality

$$
\left\|v_{h}^{n+1}\right\|_{\bar{C}} \leqslant \frac{\left\|r_{h}^{n}\right\|_{\bar{C}}+\left\|s_{h}^{n}\right\|_{\bar{C}}}{2 \rho c} \leqslant \ldots \leqslant \frac{\left\|r_{h}^{0}\right\|_{\bar{C}}+\left\|s_{h}^{0}\right\|_{\bar{C}}}{2 \rho c} \leqslant\left\|v_{h}^{0}\right\|_{\bar{C}}+\frac{\left\|p_{h}^{0}\right\|_{\bar{C}}}{\rho c}<c
$$

takes place. This completes the proof of the theorem.

In order to obtain an a priori estimate of the solution of the difference problem (4.6)(4.8) it is necessary to estimate the difference derivatives $\tilde{r}_{h \bar{x}}, \tilde{s}_{h x}$ for the perturbed solution. Differentiating the first equation (4.1) with respect to $\bar{x}$ and the second equation with respect to $x$ and introducing notations $\xi=\tilde{r}_{h \bar{x}}, \eta=\tilde{s}_{h x}$, we get

$$
\xi_{t, i}+\left(\left(\tilde{v_{h}}+c\right) \hat{\xi}\right)_{\bar{x}, i}=0, \quad i=\overline{2, N}, \quad \eta_{t, i}+\left(\left(\tilde{v_{h}}-c\right) \hat{\eta}\right)_{x, i}=0, \quad i=\overline{0, N-2} .
$$


Let us consider the first equation (4.1) for $i=1$ and the second equation for $i=N-1$. Taking into account the homogeneity of the boundary conditions $\tilde{r}_{h, 0}=0, \tilde{s}_{h, N}=0$ for the perturbed problem $(4.1)-(4.3)$, we get the boundary conditions for the difference scheme (4.11) in the form

$$
\xi_{t, 1}=-h^{-1}\left(\tilde{v}_{h, 1}+c\right) \hat{\xi}_{1}, \quad \eta_{t, N-1}=h^{-1}\left(\tilde{v}_{h, N-1}-c\right) \hat{\eta}_{N-1} .
$$

Due to inequalities (4.5) the initial conditions for the difference scheme (4.11)

$$
\begin{aligned}
& \xi_{i}^{0}=\tilde{r}_{h \bar{x}, i}^{0}=\frac{1}{h} \int_{x_{i-1}}^{x_{i}} \frac{\partial \tilde{r}}{\partial x}(x, 0) d x=\frac{1}{h} \int_{x_{i-1}}^{x_{i}} \tilde{r}_{0}^{\prime}(x) d x, \\
& \eta_{i}^{0}=\tilde{s}_{h x, i}^{0}=\frac{1}{h} \int_{x_{i}}^{x_{i}+1} \frac{\partial \tilde{s}}{\partial x}(x, 0) d x=\frac{1}{h} \int_{x_{i}}^{x_{i}+1} \tilde{s}_{0}^{\prime}(x) d x
\end{aligned}
$$

satisfy the following inequalities:

$$
\xi_{i}^{0} \geqslant 0, \quad i=\overline{1, N}, \quad \eta_{i}^{0} \leqslant 0, \quad i=\overline{0, N-1} .
$$

We write the difference scheme $(4.11)-(4.13)$ in the canonical form

$$
\begin{gathered}
A_{\xi, i}^{n} \xi_{i-1}^{n+1}-C_{\xi, i}^{n} \xi_{i}^{n+1}=-F_{\xi, i}^{n}, \quad i=\overline{2, N}, \quad \xi_{1}^{n+1}=\frac{\xi_{1}^{n}}{1+\gamma\left(\tilde{v}_{h, 1}^{n}+c\right)}, \\
-C_{\eta, i}^{n} \eta_{i}^{n+1}+B_{\eta, i}^{n} \eta_{i+1}^{n+1}=-F_{\eta, i}^{n}, \quad i=\overline{0, N-2}, \quad \eta_{N-1}^{n+1}=\frac{\eta_{N-1}^{n}}{1+\gamma\left(-\tilde{v}_{h, N-1}^{n}+c\right)},
\end{gathered}
$$

where $A_{\xi, i}^{n}=\gamma\left(\tilde{v}_{h, i-1}^{n}+c\right), C_{\xi, i}^{n}=1+\gamma\left(\tilde{v}_{h, i}^{n}+c\right), F_{\xi, i}^{n}=\xi_{i}^{n}, B_{\eta, i}^{n}=-\gamma\left(\tilde{v}_{h, i+1}^{n}-c\right)$, $C_{\eta, i}^{n}=1-\gamma\left(\tilde{v}_{h, i}^{n}-c\right), F_{\eta, i}^{n}=\eta_{i}^{n}, \gamma=\tau / h$.

The following theorem takes place.

Theorem 4.2. Let conditions (4.4), (4.5) be met. Then for the difference derivatives of the solution of the difference problem (4.1)-(4.3) the estimates

$$
\left\|\tilde{r}_{h \bar{x}}^{n}\right\|_{C^{+}} \leqslant\left\|\tilde{p}_{0 \bar{x}}+\rho c \tilde{v}_{0 \bar{x}}\right\|_{C^{+}} \leqslant c_{0}, \quad\left\|\tilde{s}_{h x}^{n}\right\|_{C^{-}} \leqslant\left\|\tilde{p}_{0 x}-\rho c \tilde{v}_{0 x}\right\|_{C^{-}} \leqslant c_{1}, \quad n=\overline{0, N_{0}}
$$

are valid.

Proof. In proving Theorem 4.1, it was shown that $\left|v_{h, i}^{n}\right|<c, i=\overline{0, N}, n=\overline{0, N_{0}}$. Similarly, by mathematical induction it is possible to prove that $\left|\tilde{v}_{h, i}^{n}\right|<c, i=\overline{0, N}, n=$ $\overline{0, N_{0}}$. Hence $A_{\xi, i}^{n}>0, B_{\eta, i}^{n}>0$.

On the first time layer $t=\tau$ we have $\tilde{v}_{h x, i}^{0}=\left(\xi_{i+1}^{0}-\eta_{i}^{0}\right) /(2 \rho c) \geqslant 0, A_{\xi, i}^{0}>0, D_{\xi, i}^{0}=$ $C_{\xi, i}^{0}-A_{\xi, i}^{0}=1+\tau \tilde{v}_{h \bar{x}, i}^{0} \geqslant 1, B_{\eta, i}^{0}>0, D_{\eta, i}^{0}=C_{\eta, i}^{0}-B_{\eta, i}^{0}=1+\tau \tilde{v}_{h x, i}^{0} \geqslant 1$. On the basis of Lemmas 3.1, 3.2 we get

$$
\begin{gathered}
\left\|\xi^{1}\right\|_{C^{+}} \leqslant \max \left\{\frac{\left|\xi_{1}^{0}\right|}{1+\gamma\left(\tilde{v}_{h, 1}^{0}+c\right)}, \max _{2 \leqslant i \leqslant N} \frac{\left|\xi_{i}^{0}\right|}{1+\tau \tilde{v}_{h \bar{x}, i}^{0}}\right\} \leqslant\left\|\xi^{0}\right\|_{C^{+}}, \\
\left\|\eta^{1}\right\|_{C^{-}} \leqslant \max \left\{\frac{\left|\eta_{N-1}^{0}\right|}{1+\gamma\left(-\tilde{v}_{h, N-1}^{0}+c\right)}, \max _{0 \leqslant i \leqslant N-2} \frac{\left|\eta_{i}^{0}\right|}{1+\tau \tilde{v}_{h x, i}^{0}}\right\} \leqslant\left\|\eta^{0}\right\|_{C^{-}} .
\end{gathered}
$$


From inequalities (4.14)

$$
\xi_{1}^{1}=\frac{\xi_{1}^{0}}{1+\gamma\left(\tilde{v}_{h, 1}^{0}+c\right)} \geqslant 0, \quad \eta_{N-1}^{1}=\frac{\eta_{N-1}^{0}}{1+\gamma\left(-\tilde{v}_{h, N-1}^{0}+c\right)} \leqslant 0
$$

follows. By virtue of the positivity of the coefficients of difference schemes (4.15), (4.16), we get $\xi_{i}^{1} \geqslant 0, i=\overline{2, N}, \eta_{i}^{1} \leqslant 0, i=\overline{0, N-2}$. Hence $\xi_{i}^{1} \geqslant 0, i=\overline{1, N}, \eta_{i}^{1} \leqslant 0, i=\overline{0, N-1}$. Therefore we have

$$
\tilde{v}_{h x, i}^{1}=\frac{\tilde{r}_{h x, i}^{1}-\tilde{s}_{h x, i}^{1}}{2 \rho c}=\frac{\xi_{i+1}^{1}-\eta_{i}^{1}}{2 \rho c} \geqslant 0, \quad i=\overline{0, N-1} .
$$

Suppose that on the time layer $n$ the conditions $\tilde{v}_{h x, i}^{n} \geqslant 0, i=\overline{0, N-1}, \xi_{i}^{n} \geqslant 0, i=\overline{1, N}$, $\eta_{i}^{n} \leqslant 0, i=\overline{0, N-1}$ are satisfied. From the induction assumption it follows that $A_{\xi, i}^{n}>0$, $D_{\xi, i}^{n}=C_{\xi, i}^{n}-A_{\xi, i}^{n}=1+\tau \tilde{v}_{h \bar{x}, i}^{n}>0, B_{\eta, i}^{n}>0, D_{\eta, i}^{n}=C_{\eta, i}^{n}-B_{\eta, i}^{0}=1+\tau \tilde{v}_{h x, i}^{n}>0$ and it is possible to show that on the layer $(n+1)$ we have $\tilde{v}_{h x, i}^{n+1} \geqslant 0, i=\overline{0, N-1}, \xi_{i}^{n+1} \geqslant 0, i=\overline{1, N}$, $\eta_{i}^{n+1} \leqslant 0, i=\overline{0, N-1}$ and for the derivatives of the perturbed solution the estimates

$$
\begin{aligned}
& \left\|\tilde{r}_{h \bar{x}}^{n+1}\right\|_{C^{+}}=\left\|\xi^{n+1}\right\|_{C^{+}} \leqslant\left\|\xi^{n}\right\|_{C^{+}} \leqslant \ldots \leqslant\left\|\xi^{0}\right\|_{C^{+}}=\left\|\tilde{p}_{0 \bar{x}}+\rho c \tilde{v}_{0 \bar{x}}\right\|_{C^{+}} \leqslant c_{0}, \\
& \left\|\tilde{s}_{h x}^{n+1}\right\|_{C^{-}}=\left\|\eta^{n+1}\right\|_{C^{-}} \leqslant\left\|\xi^{n}\right\|_{C^{-}} \leqslant \ldots \leqslant\left\|\eta^{0}\right\|_{C^{-}}=\left\|\tilde{p}_{0 x}-\rho c \tilde{v}_{0 x}\right\|_{C^{-}} \leqslant c_{1}
\end{aligned}
$$

are valid. This completes the proof of the theorem.

Theorem 4.3. Let conditions (2.4), (2.5), (4.4), (4.5) be met. Then the difference scheme (2.9)-(2.11) is $\rho$-stable in the sense of the initial data and for the solution the estimate

$$
\left\|\tilde{r}_{h}^{n+1}-r_{h}^{n+1}\right\|_{\bar{C}}+\left\|\tilde{s}_{h}^{n+1}-s_{h}^{n+1}\right\|_{\bar{C}} \leqslant e^{T\left(\tilde{c_{0}}+\tilde{c_{1}}\right)}\left(\left\|\delta p_{0}+\rho c \delta v_{0}\right\|_{\bar{C}}+\left\|\delta p_{0}-\rho c \delta v_{0}\right\|_{\bar{C}}\right)
$$

is valid, where $\tilde{c}_{0}=c_{0} /(2 \rho c), \tilde{c}_{1}=c_{1} /(2 \rho c)$.

Proof. W e write the difference scheme (4.6)-(4.8) in the canonical form

$$
\begin{gathered}
A_{r, i}^{n} \delta r_{i-1}^{n+1}-C_{r, i}^{n} \delta r_{i}^{n+1}=-F_{r, i}^{n}, \quad i=\overline{1, N}, \quad \delta r_{0}^{n+1}=0, \\
-C_{s, i}^{n} \delta s_{i}^{n+1}+B_{s, i}^{n} \delta s_{i+1}^{n+1}=-F_{s, i}^{n}, \quad i=\overline{0, N-1}, \quad \delta s_{N}^{n+1}=0,
\end{gathered}
$$

where

$$
\begin{gathered}
A_{r, i}^{n}=\gamma\left(v_{h, i}^{n}+c\right), \quad C_{r, i}^{n}=1+\gamma\left(v_{h, i}^{n}+c\right), \quad F_{r, i}^{n}=\left(1-\frac{\tau}{2 \rho c} \tilde{r}_{h \bar{x}}^{n+1}\right) \delta r_{i}^{n}+\frac{\tau}{2 \rho c} \tilde{r}_{h \bar{x}}^{n+1} \delta s_{i}^{n}, \\
B_{s, i}^{n}=-\gamma\left(v_{h, i}^{n}-c\right), \quad C_{s, i}^{n}=1-\gamma\left(v_{h, i}^{n}-c\right), \quad F_{s, i}^{n}=\left(1+\frac{\tau}{2 \rho c} \tilde{s}_{h x}^{n+1}\right) \delta s_{i}^{n}-\frac{\tau}{2 \rho c} \tilde{s}_{h x}^{n+1} \delta r_{i}^{n} .
\end{gathered}
$$

Since for the coefficients of the difference scheme the conditions of Lemmas 3.1, 3.2 are hold, then due to the obtained estimates (4.17) for the derivatives of the perturbed solution we have

$$
\begin{gathered}
\left\|\delta r^{n+1}\right\|_{\bar{C}}=\left\|\delta r^{n+1}\right\|_{C^{+}} \leqslant\left(1+\frac{\tau}{2 \rho c}\left\|\tilde{r}_{h \bar{x}}^{n+1}\right\|_{C^{+}}\right)\left\|\delta r^{n}\right\|_{C^{+}}+\frac{\tau}{2 \rho c}\left\|\tilde{r}_{h \bar{x}}^{n+1}\right\|_{C^{+}}\left\|\delta s^{n}\right\|_{C^{+}} \leqslant \\
\left(1+\frac{\tau c_{0}}{2 \rho c}\right)\left\|\delta r^{n}\right\|_{\bar{C}}+\frac{\tau c_{0}}{2 \rho c}\left\|\delta s^{n}\right\|_{\bar{C}} \leqslant\left(1+\tau \tilde{c}_{0}\right)\left\|\delta r^{n}\right\|_{\bar{C}}+\tau \tilde{c}_{0}\left\|\delta s^{n}\right\|_{\bar{C}},
\end{gathered}
$$




$$
\begin{gathered}
\left\|\delta s^{n+1}\right\|_{\bar{C}}=\left\|\delta s^{n+1}\right\|_{C^{-}} \leqslant\left(1+\frac{\tau}{2 \rho c}\left\|\tilde{s}_{h x}^{n+1}\right\|_{C^{-}}\right)\left\|\delta s^{n}\right\|_{C^{-}}+\frac{\tau}{2 \rho c}\left\|\tilde{s}_{h x}^{n+1}\right\|_{C^{-}}\left\|\delta r^{n}\right\|_{C^{-}} \leqslant \\
\left(1+\frac{\tau c_{1}}{2 \rho c}\right)\left\|\delta s^{n}\right\|_{\bar{C}}+\frac{\tau c_{1}}{2 \rho c}\left\|\delta r^{n}\right\|_{\bar{C}} \leqslant\left(1+\tau \tilde{c}_{1}\right)\left\|\delta s^{n}\right\|_{\bar{C}}+\tau \tilde{c}_{1}\left\|\delta r^{n}\right\|_{\bar{C}} .
\end{gathered}
$$

Summing estimates (4.21) and (4.22), we get

$$
\left\|\delta r^{n+1}\right\|_{\bar{C}}+\left\|\delta s^{n+1}\right\|_{\bar{C}} \leqslant\left(1+\tau\left(\tilde{c}_{0}+\tilde{c}_{1}\right)\right)\left(\left\|\delta r^{n}\right\|_{\bar{C}}+\left\|\delta s^{n}\right\|_{\bar{C}}\right) \leqslant e^{\tau\left(\tilde{c}_{0}+\tilde{c}_{1}\right)}\left(\left\|\delta r^{n}\right\|_{\bar{C}}+\left\|\delta s^{n}\right\|_{\bar{C}}\right) .
$$

Applying this inequality sequentially, we obtain

$$
\left\|\tilde{r}_{h}^{n+1}-r_{h}^{n+1}\right\|_{\bar{C}}+\left\|\tilde{s}_{h}^{n+1}-s_{h}^{n+1}\right\|_{\bar{C}} \leqslant \ldots \leqslant e^{T\left(\tilde{c}_{0}+\tilde{c}_{1}\right)}\left(\left\|\delta p_{0}+\rho c \delta v_{0}\right\|_{\bar{C}}+\left\|\delta p_{0}-\rho c \delta v_{0}\right\|_{\bar{C}}\right) .
$$

This completes the proof of the theorem.

Thus, we have proved the $\rho$-stability of the difference scheme $(2.9)-(2.11)$ for an arbitrary instant of time $T$ under conditions (2.5), (4.5). From these conditions, in particular, $v_{0}^{\prime}(x) \geqslant 0, \tilde{v}_{0}^{\prime}(x) \geqslant 0$ follows. However, independent of the smoothness of the input data the nonlinearity of the system of equations can generate various physical effects, such as "gradient catastrophe" or a shock wave [6]. In fact, conditions (2.5), (4.5) denote the absence of domains with large gradients. Therefore, of certain interest is the analysis of the stability of the difference scheme without the above assumptions. In this case, the following statement takes place.

Theorem 4.4. Let conditions (2.4), (4.4) be met. Then the difference scheme (2.9)(2.11) is $\rho$-stable in the sense of the initial data and for its solution the estimate

$$
\left\|\tilde{r}_{h}^{n+1}-r_{h}^{n+1}\right\|_{\bar{C}}+\left\|\tilde{s}_{h}^{n+1}-s_{h}^{n+1}\right\|_{\bar{C}} \leqslant e^{T c_{2} /(2 \rho c)}\left(\left\|\delta p_{0}+\rho c \delta v_{0}\right\|_{\bar{C}}+\left\|\delta p_{0}-\rho c \delta v_{0}\right\|_{\bar{C}}\right)
$$

is valid for $T<t_{0}$, where $t_{0}=1 /\left[(\rho c)^{-1}\left\|p_{\bar{x}}^{0}\right\|_{C^{+}}+\left\|v_{\bar{x}}^{0}\right\|_{C^{+}}\right]$.

Proof. Consider now problem $(2.1)-(2.4)$. In this case, the sign of the derivative $\left(v^{0}(x)\right)^{\prime}$ is unknown. Let us introduce into the consideration the norm $Q^{n}=\left(\left\|\xi^{n}\right\|_{C^{+}}+\left\|\eta^{n}\right\|_{C^{-}}\right) / 2 \rho c$. Let $1-t_{n} Q^{0}>0, n=1,2, \ldots, N_{0}$. Then on the first time layer $t_{1}=\tau$ we have $1+\tau \tilde{v}_{h \bar{x}, i}^{0} \geqslant$ $1-\tau\left\|\tilde{v}_{h \bar{x}}^{0}\right\|_{C^{+}} \geqslant 1-\tau Q^{0}>0, i=\overline{1, N}$. Hence for the coefficients of the difference scheme $(4.15)-(4.16)$ the conditions of Lemmas 3.1, 3.2 are fulfilled and for the first derivatives $\tilde{r}_{h \bar{x}}^{1}$, $\tilde{s}_{h x}^{1}$ of the solution of the difference scheme $(4.1)-(4.3)$ the estimates

$$
\begin{gathered}
\left\|\xi^{1}\right\|_{C^{+}} \leqslant \max \left\{\frac{\left|\xi_{1}^{0}\right|}{1+\gamma\left(\tilde{v}_{h, 1}^{0}+c\right)}, \max _{2 \leqslant i \leqslant N} \frac{\left|\xi_{i}^{0}\right|}{1+\tau \tilde{v}_{h \bar{x}, i}^{0}}\right\} \leqslant \max \left\{\frac{\left|\xi_{1}^{0}\right|}{1+\tau \tilde{v}_{h \bar{x}, 1}^{0}}, \max _{2 \leqslant i \leqslant N} \frac{\left|\xi_{i}^{0}\right|}{1+\tau \tilde{v}_{h \bar{x}, i}^{0}}\right\} \leqslant \\
\max _{1 \leqslant i \leqslant N} \frac{\left|\xi_{i}^{0}\right|}{1-\tau\left|\tilde{v}_{h \bar{x}, i}^{0}\right|} \leqslant \frac{\left\|\xi^{0}\right\|_{C^{+}}}{1-\frac{\tau}{2 \rho c}\left(\left\|\xi^{0}\right\|_{C^{+}}+\left\|\eta^{0}\right\|_{C^{-}}\right)}, \\
\left\|\eta^{1}\right\|_{C^{-}} \leqslant \max \left\{\frac{\left|\eta_{N-1}^{0}\right|}{1+\gamma\left(-\tilde{v}_{h, N-1}^{0}+c\right)}, \max _{0 \leqslant i \leqslant N-2} \frac{\left|\eta_{i}^{0}\right|}{1+\tau \tilde{v}_{h x, i}^{0}}\right\} \leqslant
\end{gathered}
$$

$\max \left\{\frac{\left|\eta_{N-1}^{0}\right|}{1+\tau \tilde{v}_{h x, N-1}^{0}}, \max _{0 \leqslant i \leqslant N-2} \frac{\left|\eta_{i}^{0}\right|}{1+\tau \tilde{v}_{h x, i}^{0}}\right\} \leqslant \max _{0 \leqslant i \leqslant N-1} \frac{\left|\eta_{i}^{0}\right|}{1-\tau\left|\tilde{v}_{h x, i}^{0}\right|} \leqslant \frac{\left\|\eta^{0}\right\|_{C^{-}}}{1-\frac{\tau}{2 \rho c}\left(\left\|\xi^{0}\right\|_{C^{+}}+\left\|\eta^{0}\right\|_{C^{-}}\right)}$

take place. Summing the obtained estimates, we have

$$
Q^{1} \leqslant \frac{Q^{0}}{1-\tau Q^{0}}
$$


Then

$$
1+\tau \tilde{v}_{h \bar{x}, i}^{1} \geqslant 1-\tau\left\|\tilde{v}_{h \bar{x}}^{1}\right\|_{C^{+}} \geqslant 1-\tau Q^{1} \geqslant 1-\frac{\tau Q^{0}}{1-\tau Q^{0}}=\frac{1-t_{2} Q^{0}}{1-t_{1} Q^{0}}>0 .
$$

By the mathematical induction method it is possible to show the fulfillment of inequalities

$$
Q^{n} \leqslant \frac{Q^{n-1}}{1-\tau Q^{n-1}}, \quad n=1,2, \ldots, N_{0}
$$

and on the basis of Lemma 3.3 for the first derivatives of the solution of the difference scheme $(4.1)-(4.3)$ the estimate

$$
\left\|\xi^{n+1}\right\|_{C^{+}}+\left\|\eta^{n+1}\right\|_{C^{-}} \leqslant \frac{\left\|\xi^{0}\right\|_{C^{+}}+\left\|\eta^{0}\right\|_{C^{-}}}{1-\frac{t^{n+1}}{2 \rho c}\left(\left\|\xi^{0}\right\|_{C^{+}}+\left\|\eta^{0}\right\|_{C^{-}}\right)} \leqslant \frac{2\left\|\tilde{p}_{0 \bar{x}}\right\|_{C^{+}}+2 \rho c\left\|\tilde{v}_{0 \bar{x}}\right\|_{C^{+}}}{1-t^{n}\left(\frac{1}{\rho c}\left\|p_{0 \bar{x}}\right\|_{C^{+}}+\left\|v_{0 \bar{x}}\right\|_{C^{+}}\right)}=c_{2}
$$

takes place.

Therefore, the derivatives $\tilde{r}_{h \bar{x}}^{n}, \tilde{s}_{h x}^{n}$ are bounded according to the norm $Q$ only for the time instant $t_{n}<t_{0}$, where

$$
t_{0}=\frac{1}{\frac{1}{\rho c}\left\|p_{\bar{x}}^{0}\right\|_{C^{+}}+\left\|v_{\bar{x}}^{0}\right\|_{C^{+}}} .
$$

Summing the estimates for perturbations

$$
\begin{gathered}
\left\|\delta r^{n+1}\right\|_{\bar{C}}=\left\|\delta r^{n+1}\right\|_{C^{+}} \leqslant\left(1+\frac{\tau}{2 \rho c}\left\|\tilde{r}_{h \bar{x}}^{n+1}\right\|_{C^{+}}\right)\left\|\delta r^{n}\right\|_{C^{+}}+\frac{\tau}{2 \rho c}\left\|\tilde{r}_{h \bar{x}}^{n+1}\right\|_{C^{+}}\left\|\delta s^{n}\right\|_{C^{+}}, \\
\left\|\delta s^{n+1}\right\|_{\bar{C}}=\left\|\delta s^{n+1}\right\|_{C^{-}} \leqslant\left(1+\frac{\tau}{2 \rho c}\left\|\tilde{s}_{h x}^{n+1}\right\|_{C^{-}}\right)\left\|\delta s^{n}\right\|_{C^{-}}+\frac{\tau}{2 \rho c}\left\|\tilde{s}_{h x}^{n+1}\right\|_{C^{-}}\left\|\delta r^{n}\right\|_{C^{-}}
\end{gathered}
$$

we obtain the inequality

$$
\begin{gathered}
\left\|\delta r^{n+1}\right\|_{\bar{C}}+\left\|\delta s^{n+1}\right\|_{\bar{C}} \leqslant\left(1+\frac{\tau}{2 \rho c}\left(\left\|\tilde{r}_{h \bar{x}}^{n+1}\right\|_{C^{-}}+\left\|\tilde{s}_{h x}^{n+1}\right\|_{C^{+}}\right)\right) \times \\
\left(\left\|\delta r^{n}\right\|_{\bar{C}}+\left\|\delta s^{n}\right\|_{\bar{C}}\right) \leqslant e^{\tau c_{2} /(2 \rho c)}\left(\left\|\delta r^{n}\right\|_{\bar{C}}+\left\|\delta s^{n}\right\|_{\bar{C}}\right) .
\end{gathered}
$$

Applying this inequality sequentially, we get

$$
\left\|\tilde{r}_{h}^{n+1}-r_{h}^{n+1}\right\|_{\bar{C}}+\left\|\tilde{s}_{h}^{n+1}-s_{h}^{n+1}\right\|_{\bar{C}} \leqslant \ldots \leqslant e^{T c_{2} /(2 \rho c)}\left(\left\|\delta p_{0}+\rho c \delta v_{0}\right\|_{\bar{C}}+\left\|\delta p_{0}-\rho c \delta v_{0}\right\|_{\bar{C}}\right) .
$$

This completes the proof of the theorem.

When $t \geqslant t_{n} \geqslant t_{0}$, then the derivatives become unlimited and it is impossible to prove the difference scheme stability for $t \geqslant t_{n}$.

Remark 4.1. Much in the same manner, the uniqueness of the solution of the nonlinear difference scheme $(2.9)-(2.11)$ is proved. The proof is carried out by contradiction. We assume that there exist two solutions $\left(r_{1 h}, s_{1 h}\right),\left(r_{2 h}, s_{2 h}\right)$ that satisfy the difference scheme with the same initial and boundary conditions. Then for the differences $R=r_{2 h}-r_{1 h}, S=$ $s_{2 h}-s_{1 h}$ we get the following system of difference equations with homogeneous initial and boundary conditions:

$$
R_{t, i}+\left(v_{1 h, i}+c\right) \hat{R}_{\bar{x}, i}+\hat{r}_{2 h \bar{x}, i} \frac{R_{i}}{2 \rho c}-\hat{r}_{2 h \bar{x}, i} \frac{S_{i}}{2 \rho c}=0, \quad i=\overline{1, N}
$$




$$
\begin{gathered}
S_{t, i}+\left(v_{1 h, i}-c\right) \hat{S}_{x, i}+\hat{s}_{2 h x, i} \frac{R_{i}}{2 \rho c}-\hat{s}_{2 h x, i} \frac{S_{i}}{2 \rho c}=0, \quad i=\overline{0, N-1} \\
\hat{R}_{0}=0, \quad \hat{S}_{N}=0, \quad R_{i}^{0}=0, \quad S_{i}^{0}=0, \quad i=\overline{1, N} .
\end{gathered}
$$

Using the investigation technique from this section, we can get estimates of the kind

$$
\left\|r_{2 h}^{n+1}-r_{1 h}^{n+1}\right\|_{\bar{C}}+\left\|s_{2 h}^{n+1}-s_{1 h}^{n+1}\right\|_{\bar{C}} \leqslant 0 .
$$

Therefore, the difference scheme has a unique solution.

\section{Investigation of the convergence}

In investigating the convergence of the difference scheme $(2.9)-(2.11)$, we will suppose that for the solutions $r(x, t), s(x, t)$ of the differential problem there exist continuous second-order derivatives with respect to the space and time: $r(x, t) \in C^{2,2}\left(\bar{Q}_{T}\right), s(x, t) \in C^{2,2}\left(\bar{Q}_{T}\right)$. Now we introduce the errors of the method $\Delta r=r_{h}-r, \Delta s=s_{h}-s$. Substituting $r_{h}=\Delta r+r$, $s_{h}=\Delta s+s$ into equations $(2.9)-(2.11)$, we arrive at the following problem:

$$
\begin{gathered}
\Delta r_{t, i}+\left(v_{h, i}+c\right) \Delta \hat{r}_{\bar{x}, i}+\left(\frac{\Delta r_{i}-\Delta s_{i}}{2 \rho c}\right) \hat{r}_{\bar{x}, i}=\psi_{1, i}, \quad i=\overline{1, N} \\
\Delta s_{t, i}+\left(v_{h, i}-c\right) \Delta \hat{s}_{x, i}+\left(\frac{\Delta r_{i}-\Delta s_{i}}{2 \rho c}\right) \hat{s}_{x, i}=\psi_{2, i}, \quad i=\overline{0, N-1} \\
\Delta r(x, 0)=0, \quad \Delta s(x, 0)=0, \quad x \in \bar{\omega}_{h}, \\
\Delta r(0, \hat{t})=0, \quad \Delta s(l, \hat{t})=0, \quad \hat{t} \in \omega_{\tau}, \\
\psi_{1}=-r_{t}-(v+c) \hat{r}_{\bar{x}}=\left(\frac{\partial r}{\partial t}-r_{t}\right)+\left((v+c) \frac{\partial r}{\partial x}-(v+c) \hat{r}_{\bar{x}}\right) \\
\psi_{2}=-s_{t}-(v-c) \hat{s}_{x}=\left(\frac{\partial s}{\partial t}-s_{t}\right)+\left((v-c) \frac{\partial s}{\partial x}-(v-c) \hat{s}_{x}\right) .
\end{gathered}
$$

Using the Tailor series expansion, it is easy to show that the errors of the approximation $\psi_{1}, \psi_{2}$ satisfy the following relation:

$$
\max _{t \in \omega_{\tau}}\left\|\psi_{1}(t)\right\|_{\bar{C}}+\max _{t \in \omega_{\tau}}\left\|\psi_{2}(t)\right\|_{\bar{C}} \leqslant c_{3}(h+\tau), \quad c_{3}=\text { const }>0
$$

We now write the difference scheme (5.1) - (5.4) in the canonical form

$$
\begin{gathered}
A_{r, i}^{n} \Delta r_{i-1}^{n+1}-C_{r, i}^{n} \Delta r_{i}^{n+1}=-F_{r, i}^{n}, \quad i=\overline{1, N}, \quad \Delta r_{0}^{n+1}=0, \\
-C_{s, i}^{n} \Delta s_{i}^{n+1}+B_{s, i}^{n} \Delta s_{i+1}^{n+1}=-F_{s, i}^{n}, \quad i=\overline{0, N-1}, \quad \Delta s_{N}^{n+1}=0,
\end{gathered}
$$

where

$$
\begin{aligned}
& A_{r, i}^{n}=\gamma\left(v_{h, i}^{n}+c\right), \quad C_{r, i}^{n}=1+\gamma\left(v_{h, i}^{n}+c\right), \quad F_{r, i}^{n}=\left(1-\frac{\tau}{2 \rho c} r_{\bar{x}}^{n+1}\right) \Delta r_{i}^{n}+\frac{\tau}{2 \rho c} r_{\bar{x}}^{n+1} \Delta s_{i}^{n}+\tau \psi_{1, i}^{n}, \\
& B_{s, i}^{n}=-\gamma\left(v_{h, i}^{n}-c\right), \quad C_{s, i}^{n}=1-\gamma\left(v_{h, i}^{n}-c\right), \quad F_{s, i}^{n}=\left(1+\frac{\tau}{2 \rho c} s_{x}^{n+1}\right) \Delta s_{i}^{n}-\frac{\tau}{2 \rho c} s_{x}^{n+1} \Delta r_{i}^{n}+\tau \psi_{2, i}^{n} .
\end{aligned}
$$


As was shown in Section 4 , the inequality $\left|v_{h}^{n}\right|<c$ holds. Then for the coefficients of the difference scheme (5.5), (5.6) the conditions of Lemmas 3.1, 3.2 hold. Therefore,

$$
\begin{gathered}
\left\|\Delta r^{n+1}\right\|_{\bar{C}}=\left\|\Delta r^{n+1}\right\|_{C^{+}} \leqslant\left(1+\frac{\tau}{2 \rho c}\left\|r_{\bar{x}}^{n+1}\right\|_{C^{+}}\right)\left\|\Delta r^{n}\right\|_{C^{+}}+\frac{\tau}{2 \rho c}\left\|r_{\bar{x}}^{n+1}\right\|_{C^{+}}\left\|\Delta s^{n}\right\|_{C^{+}+} \\
\tau\left\|\psi_{1}^{n}\right\|_{\bar{C}} \leqslant\left(1+\frac{\tau c_{4}}{2 \rho c}\right)\left\|\Delta r^{n}\right\|_{\bar{C}}+\frac{\tau c_{4}}{2 \rho c}\left\|\Delta s^{n}\right\|_{\bar{C}}+\tau\left\|\psi_{1}^{n}\right\|_{\bar{C}} \leqslant \\
\left(1+\tau \tilde{c_{4}}\right)\left\|\Delta r^{n}\right\|_{\bar{C}}+\tau \tilde{c_{4}}\left\|\Delta s^{n}\right\|_{\bar{C}}+\tau\left\|\psi_{1}^{n}\right\|_{\bar{C}}, \\
\left\|\Delta s^{n+1}\right\|_{\bar{C}}=\left\|\Delta s^{n+1}\right\|_{C^{-}} \leqslant\left(1+\frac{\tau}{2 \rho c}\left\|s_{x}^{n+1}\right\|_{C^{-}}\right)\left\|\Delta s^{n}\right\|_{C^{-}}+\frac{\tau}{2 \rho c}\left\|s_{x}^{n+1}\right\|_{C^{-}}\left\|\Delta r^{n}\right\|_{C^{-}} \\
\tau\left\|\psi_{2}^{n}\right\|_{\bar{C}} \leqslant\left(1+\frac{\tau c_{5}}{2 \rho c}\right)\left\|\Delta s^{n}\right\|_{\bar{C}}+\frac{\tau c_{5}}{2 \rho c}\left\|\delta r^{n}\right\|_{\bar{C}}+\tau\left\|\psi_{2}^{n}\right\|_{\bar{C}} \leqslant \\
\left(1+\tau \tilde{c}_{5}\right)\left\|\Delta s^{n}\right\|_{\bar{C}}+\tau \tilde{c_{5}}\left\|\delta r^{n}\right\|_{\bar{C}}+\tau\left\|\psi_{2}^{n}\right\|_{\bar{C}},
\end{gathered}
$$

where $c_{4}=\max _{(x, t) \in \bar{Q}_{T}}|\partial r / \partial x|, \tilde{c}_{4}=c_{4} /(2 \rho c), c_{5}=\max _{(x, t) \in \bar{Q}_{T}}|\partial s / \partial x|, \tilde{c}_{5}=c_{5} /(2 \rho c)$.

Summing estimates (5.7) and (5.8), we get

$$
\begin{gathered}
\left\|\Delta r^{n+1}\right\|_{\bar{C}}+\left\|\Delta s^{n+1}\right\|_{\bar{C}} \leqslant\left(1+\tau\left(\tilde{c_{4}}+\tilde{c_{5}}\right)\right)\left(\left\|\Delta r^{n}\right\|_{\bar{C}}+\left\|\Delta s^{n}\right\|_{\bar{C}}\right)+\tau\left(\left\|\psi_{1}^{n}\right\|_{\bar{C}}+\left\|\psi_{2}^{n}\right\|_{\bar{C}}\right) \leqslant \\
e^{\tau\left(\tilde{c_{4}}+\tilde{c_{5}}\right)}\left(\left\|\Delta r^{n}\right\|_{\bar{C}}+\left\|\Delta s^{n}\right\|_{\bar{C}}\right)+\tau\left(\left\|\psi_{1}^{n}\right\|_{\bar{C}}+\left\|\psi_{2}^{n}\right\|_{\bar{C}}\right) \leqslant \\
\sum_{k=0}^{n} \tau e^{t_{n-k}\left(\tilde{c_{4}}+\tilde{c_{5}}\right)}\left(\left\|\psi_{1}^{k}\right\|_{\bar{C}}+\left\|\psi_{2}^{k}\right\|_{\bar{C}}\right) \leqslant c_{6} t_{n+1}(h+\tau) .
\end{gathered}
$$

Thus, the following theorem takes place.

Theorem 5.1. Let the solution $r(x, t), s(x, t) \in C^{2,2}\left(\bar{Q}_{T}\right)$ of problem (2.6)-(2.8) exist, then the solution of the difference scheme (2.9)-(2.11) converges to the solution of the differential problem (2.6)-(2.8) and the following a priory estimate

$$
\left\|r_{h}^{n+1}-r^{n+1}\right\|_{\bar{C}}+\left\|s_{h}^{n+1}-s^{n+1}\right\|_{\bar{C}} \leqslant c_{6} t_{n+1}(h+\tau)
$$

holds. Here the constant $c_{6}>0$ is independent of $h, \tau$ and the approximate solution.

\section{Computating experiment}

The results confirming the theoretical conclusions are given below. As a test problem, we considered the system of differential equations (2.1) with homogeneous $\mu_{1}(t)=\mu_{2}(t)=0$ boundary conditions (2.3) and with the following initial conditions:

$$
\begin{array}{r}
v_{0}(x)=-A \cos (\pi x), \quad p_{0}(x)=A \rho c^{2}, \quad A=0.9, \quad \rho=c=1, \quad 0 \leqslant x \leqslant 1, \\
v_{0}(x)=0, \quad p_{0}(x)=A \rho c^{2} \sin (\pi x), \quad A=2.5, \quad \rho=c=1, \quad 0 \leqslant x \leqslant 1 .
\end{array}
$$

Notice that the initial data (6.1) satisfy the obtained sufficient stability conditions (2.4), (2.5), while (6.2) do not.

As evident from Fig. 6.1, under conditions (2.4), (2.5) the supersonic flows don't appear during the calculation and the solution of the difference scheme $(2.9)-(2.11)$ is stable. When 
the sufficient stability conditions are violated, unstable solutions can occur, since during the calculation the fluid velocity exceeds the sonic velocity in absolute value $\left|v_{h}\right|>c$. In this case, the statement of boundary condition become ill-posed [6]. The corresponding initial data and the results of the computating experiment are shown in Fig. 6.2.
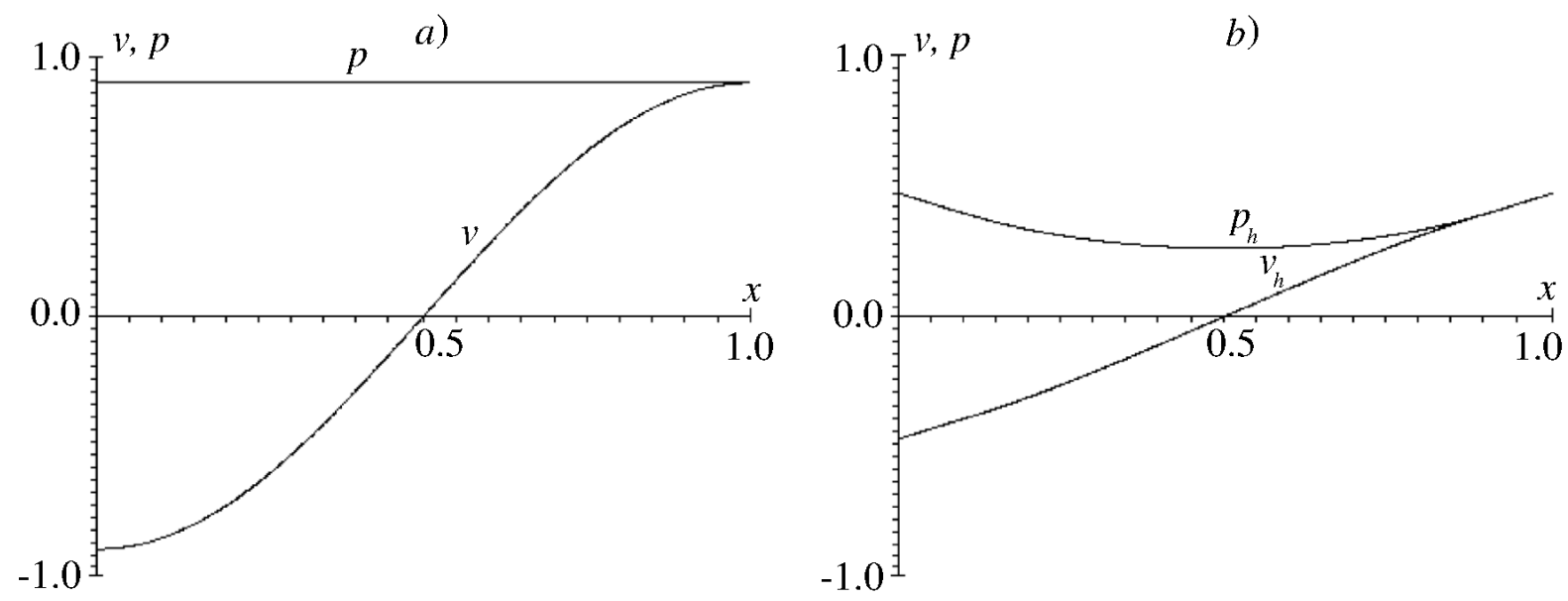

Fig. 6.1. Velocity and pressure profile lines of problem (6.1) at the initial instant of time $t=0(a)$; stable solution of the difference scheme $(2.9)-(2.11)$ with the initial data $(6.1)$ at $t=0.698(b)$
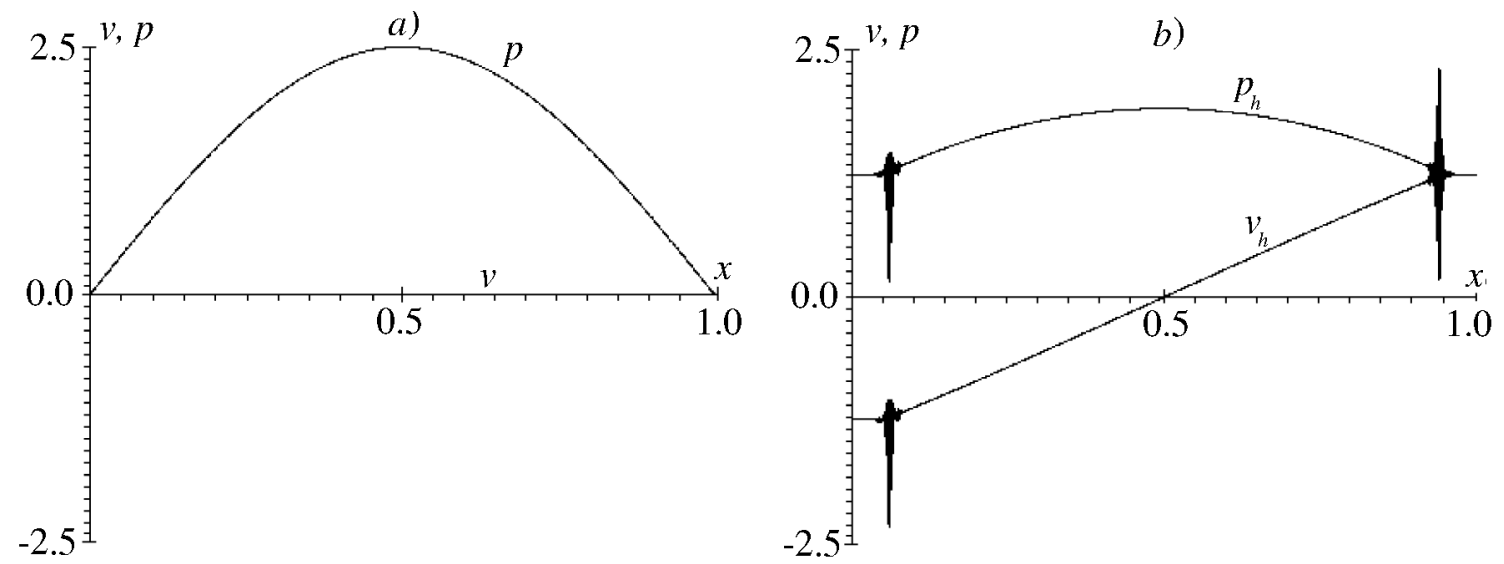

Fig. 6.2. Velocity and pressure profile lines of problem (6.2) at the initial instant of time $t=0(a)$; unstable solution of the difference scheme $(2.9)-(2.11)$ with the initial data (6.2) at $t=0.553(b)$

Now consider the following initial conditions:

$v_{0}(x)=A c \cos (3 \pi x), \quad p_{0}(x)=A \rho c^{2}, \quad A=-0.99, \quad \rho=1, \quad c=2, \quad 0 \leqslant x \leqslant 1$.

The initial data (6.3) satisfy the obtained sufficient stability condition (2.4) and don't satisfy condition (2.5).

The violation of condition (2.5) leads to the appearance of domains with large gradients (Fig. 6.3,b).

\section{Conclusions}

In the present work, the stability of the difference scheme for the equations of a weakly compressible liquid in the Riemann invariants has been investigated. The fundamental difference of the investigation of the stability in the nonlinear case is due to the necessity of additional 
investigation of the properties of the solution (proof of the existence) and of all difference derivatives entering into the nonlinear part of the difference equations.

A priory estimates of the difference scheme stability in the sense of the initial data have been obtained. The conditions imposed on the initial data to provide a subsonic flow are given. The violation of the conditions obtained leads to supersonic flows or to the appearance of domains with large gradients. The conditions imposed on the input data permit exact determination of the time of destruction of the solution of the initial boundary value problem.

a)

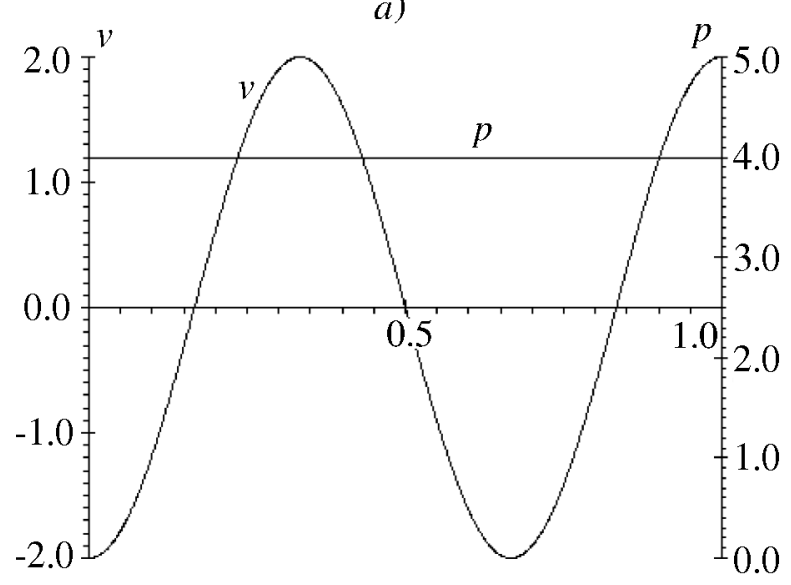

b)

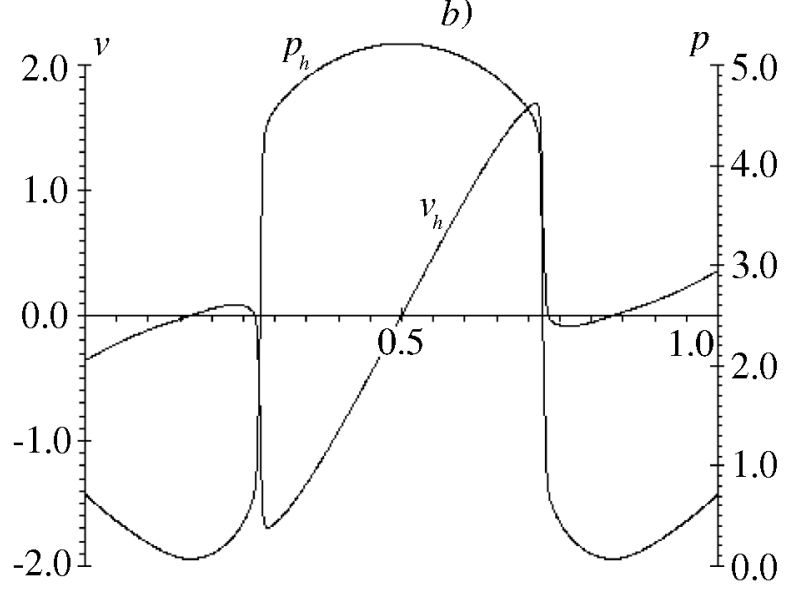

Fig. 6.3 Velocity and pressure profile lines of problem (6.3) at the initial instant of time $t=0(a)$; solution of the difference scheme (2.9)-(2.11) with the initial data (6.3) at $t=0.25(b)$

The uniqueness of the numerical solution has been proved. The investigation of the uniqueness is completely similar to the stability investigation. The convergence of the numerical solution to the solution of the differential problem the first order of accuracy in space and time has been proved.

The results of the computating experiment have confirmed the theoretical results.

\section{References}

1. A. A. Samarskii, The Theory of Difference Schemes, Marcel Dekker, Inc., New York - Basel, 2001.

2. A. A. Samarskii and A. V. Goolin, Stability of Difference Schemes, Nauka, Moscow, 1973.

3. P. Matus, Stability of difference schemes for nonlinear time-dependent problems, Comp. Meth. Appl. Math., 3 (2003), no. 2, pp. 313-329.

4. P.P. Matus and G. L. Martsynkevich, On the stability of a monotone difference scheme for the Burgers equations, Differ. Equations, 41 (2005), no. 7, pp. 1003-1009.

5. R. Temam, Navier-Stokes Equations. Theory and Numerical Analysis, 2nd ed., North-Holand, Amsterdam, 1984.

6. S. K. Godunov, Equations of Mathematical Physics, Nauka, Moscow, 1979.

7. B. L. Rozhdestvenskii and N. N. Janenko, Systems of Quasilinear Equations, Nauka, Moscow, 1968. 